\title{
Erratum to: Evaluation of biases present in the cohort multiple randomised controlled trial design: a simulation study
}

Jane Candlish ${ }^{1,2^{*}}$, Alexander Pate ${ }^{1}$, Matthew Sperrin ${ }^{1}$, Tjeerd van Staa ${ }^{1,3}$ and on behalf of GetReal Work Package 2

\section{Erratum}

After publication of the original article [1], it came to the authors' attention that there was an error affecting the References. The published Reference 14 [2] is incorrect, and should have cited a different article by Pate et al. [3].

Pate et al. [3] should have been included in the Reference list as 14 , and the subsequent references (and citations within the article text) should have been renumbered.

\section{Author details}

'Health eResearch Centre, Farr Institute for Health Informatics Research, University of Manchester, Vaughan House, Portsmouth Road, Manchester M13 9PL, UK. ${ }^{2}$ School of Health and Related Research, University of Sheffield, 30 Regent St, Sheffield S1 4DA, UK. ${ }^{3}$ Utrecht Institute for Pharmaceutical Sciences, Utrecht University, Utrecht, The Netherlands.

Published online: 22 March 2017

\section{References}

1. Candlish J, Pate A, Sperrin M, van Staa T, GetReal Work Package 2 Evaluation of biases present in the cohort multiple randomised controlled trial design: a simulation study. BMC Med Res Methodol. 2017;17:17. doi:10.1186/s12874-017-0295-7.

2. Wu J, Zhu S, Yao GL, Mohammed MA, Marshall T. Patient factors influencing the prescribing of lipid lowering drugs for primary prevention of cardiovascular disease in UK general practice: a national retrospective cohort study. PLoS One. 2013;8(7):1-10.

3. Pate A, Candlish J, Sperrin M, Van Staa TP. Cohort Multiple Randomised Controlled Trials ( $\mathrm{CmRCT}$ ) design: efficient but biased? A simulation study to evaluate the feasibility of the Cluster $\mathrm{CMRCT}$ design. BMC Med Res Meth. 2016;16:109.

\footnotetext{
* Correspondence: jane.candlish@sheffield.ac.uk

${ }^{1}$ Health eResearch Centre, Farr Institute for Health Informatics Research, University of Manchester, Vaughan House, Portsmouth Road, Manchester M13 9PL, UK

${ }^{2}$ School of Health and Related Research, University of Sheffield, 30 Regent St, Sheffield S1 4DA, UK
} 